\title{
Mixed Logic Dynamical Modeling and On Line Optimal Control of Biped Robot
}

\author{
Yingiie Yin and Shigeyuki Hosoe \\ Bio-mimetic Control Research Center, the Institute of Physical and Chemical Research \\ Japan
}

\section{Introduction}

Walking control of biped robots is classically performed in two steps: (i) the synthesis of walking patterns (Chevallereau et al., 1998) and (ii) the control of the robot to track the prescribed trajectory (Chew \& Pratt, 2000; Park \& Chung, 2000). The problems of these approaches are that the movements are non-adaptable to environment, and unexpected events are not pre-computable. In this meaning, despite the huge amount of works on the topic, biped control is still inefficient especially in terms of adaptability, robustness and stability. Contrast with this, human being decides his path on line adaptively according to the environment rather than predicting it a very long time ahead. A consideration inspired by human behavior is to introduce the path changes on line while guarantee stability and motion in environment. The automation of biped robots is very difficult but challenging.

The control problem of on line walking adaptation has been studied by some researchers. For instance, the zero moment point (ZMP) was controlled for preserving the dynamic equilibrium (Park \& Chung, 1999). However, this adaptation can only involve small changes $\varepsilon^{\prime}$ of the original trajectory. Different with this, a trajectory better adapted to the encountered \& situation is chosen on line between a set of stored trajectories (Denk \& Schmidt, 2001). The correspondent problem is that switching from one trajectory to another may lead to б unexpected effects in control. To cope with this problem, a continuous set of parameterized D trajectories was used as the candidate of choice (Chevallereau \& Sardain, 2000). By it the I. switches were suppressed, but the set of trajectories has to be defined beforehand.

3 Different with the previous considerations, a notable approach is to adopt the model predictive control (MPC) for walking adaptation (Azevedo et al., 2004; Kooij et al., 2003). By 0 optimizing on line the joint motion over a receding horizon, biped robots have been IJ controlled without pre-computed reference trajectory and switches at the algorithm level. T. The walking can auto-adapts to environment changes. However, in (Kooij et al., 2003), त accompanying with the approaching of collision, the length of the optimization horizon is o shortened step by step within the single support motion phase and finally the landing \& motion is controlled with only one sampling period optimization. The intendment of the authors is to avoid the difficulty of control when impact phenomenon occurs within the optimization horizon. But consequently, the adaptability to environment is weakened. In $\underset{\varnothing}{\varnothing}$ (Azevedo et al., 2004), how to predict the occurrence of impact then compensate positively for the effect of impact by MPC is not stated clearly. 
A model of biped walker should encapsulate phases of continuous motion, switching between types of motion, and occurrence of impacts. The overall system is naturally a hybrid one. In this research, we provide a unified modeling framework for the biped motion from the hybrid system point of view, model the biped movement as a mixed logic dynamical (MLD) system by emphasizing impact and its effect on the walking dynamics. Based on the MLD model, we adapt MPC approach to the on line walking control of biped robot. The MPC of a MLD system can be solved using powerful mixed integer quadric programming (MIQP) algorithm. Its solution corresponds to the objective-oriented optimization of the gaits. Simulation results validate the effectiveness of our proposal.

\section{Modeling of planar biped robots}

The robot is comprised of rigid links, connected by revolute joints shown in Fig.1. The model considered is a planar open kinematic chain connected at the hip joint, comprising two identical legs. Torque is applied at hip joint and between the stance leg and the support foot.

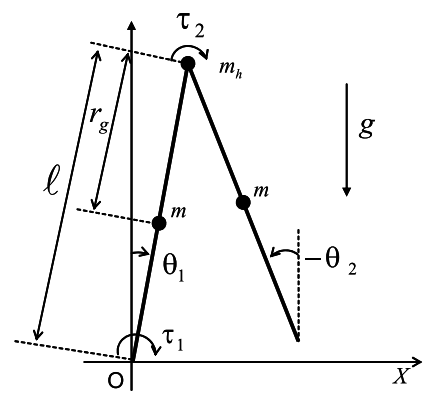

Figure 1. Coordinate of a planar biped robot. $X$ is the progressing direction. The vertical axis is in a parallel but opposite direction with gravitaty $g \cdot \theta_{1}, \theta_{2}$ is the angle of link 1 , link 2 from the vertical axis. $\tau_{1}$ and $\tau_{2}$ are the torque for driving joint 1 and joint $2 . m_{h}$ is the mass of hip, $m$ is the mass of link 1 and $2, \ell$ is the length of link 1 and $2, r_{g}$ is the distance between hip and the center of mass of each link, respectively

Motions are assumed to take place in the sagittal plane, consist of successive phases of single support and collision event, without slip. The dynamics of feet is neglected. The two phases of the walking cycle naturally lead to a mathematical model of the biped walker consisting of two parts: the differential equation describing the dynamics during the single support phase, and a velocity discontinuity caused of the impact phase. The model equations and its basic features are describing in the following.

\subsection{Dynamic equation of swing phase}

In the single support phase, one leg is pivoted to the ground while the other is swinging in the forward direction. The dynamic equation of the biped robot during the swing phase can be derived to be

$$
M(\theta) \ddot{\theta}+C(\theta, \dot{\theta}) \dot{\theta}+g(\theta)=\tau
$$


where $\theta=\left[\begin{array}{ll}\theta_{1} & \theta_{2}\end{array}\right]^{T}$ is the joint angular vector,

$$
\begin{gathered}
M(\theta)=\left[\begin{array}{cc}
m_{h} \ell^{2}+m \ell^{2}+m\left(\ell-r_{g}\right)^{2} & -m \ell r_{g} \cos \left(\theta_{1}-\theta_{2}\right) \\
-m \ell r_{g} \cos \left(\theta_{1}-\theta_{2}\right) & m r_{g}^{2}
\end{array}\right], \\
C(\theta, \dot{\theta})=\left[\begin{array}{cc}
0 & -m \ell r_{g} \sin \left(\theta_{1}-\theta_{2}\right) \dot{\theta}_{2} \\
m \ell r_{g} \sin \left(\theta_{1}-\theta_{2}\right) \dot{\theta}_{1} & 0
\end{array}\right], \\
g(\theta)=\left[\begin{array}{c}
-\left(m_{h} \ell+m\left(2 \ell-r_{g}\right)\right) g_{0} \sin \theta_{1} \\
m r_{g} g_{0} \sin \theta_{2}
\end{array}\right] .
\end{gathered}
$$

The parameter $g_{0}$ is the gravitational acceleration.

In general, the control variable is bounded because of the power limit of actuator,

$$
m_{\tau} \leq \tau \leq M_{\tau},
$$

where $m_{\tau}, M_{\tau}$ are the lower bound, the upper bound of $\tau$, respectively.

\subsection{Impact model}

For simplicity, the collision between the swing leg and the ground is assumed to be inelastic. The contact phenomenon produces two simultaneous events: 1) impact, which causes a discontinuity in the joint angular velocities, with the configuration remaining continuous, and 2) switching due to the transfer of pivot to the point of contact.

Following the law of conservation of angular momentum, we obtain a transition equation of the angular velocity as

$$
\dot{\theta}^{+}=\lambda(\alpha) \dot{\theta}^{-},
$$

where $\dot{\theta}^{+}, \dot{\theta}^{-}$denote the angular velocity of post- impact and pre-impact, respectively. $\alpha$ is the half inter-leg angle at the transition instant,

$$
\alpha=\frac{\theta_{1}^{-}-\theta_{2}^{-}}{2}=\frac{\theta_{2}^{+}-\theta_{1}^{+}}{2}>0 .
$$

The reduction ratio of velocity of the motion transmission, $\lambda(\alpha)$, is

$$
\begin{gathered}
\lambda(\alpha)=\left(Q^{+}(\alpha)\right)^{-1} Q^{-}(\alpha), \\
Q^{+}(\alpha)=\left[\begin{array}{cc}
\left(m_{h}+m\right) \ell^{2}+m\left(\ell-r_{g}\right)^{2}-m \ell r_{g} \cos (2 \alpha) & m \ell^{2}-m \ell r_{g} \cos (2 \alpha) \\
-m \ell r_{g} \cos (2 \alpha) & m r_{g}^{2}
\end{array}\right], \\
Q^{-}(\alpha)=\left[\begin{array}{cc}
\left(m_{h} \ell^{2}+2 m \ell\left(\ell-r_{g}\right)\right) \cos (2 \alpha)-m r_{g}\left(\ell-r_{g}\right) & -m r_{g}\left(\ell-r_{g}\right) \\
-m r_{g}\left(\ell-r_{g}\right) & 0
\end{array}\right] .
\end{gathered}
$$


The switching of the pivot to the point of contact is described as

$$
\theta^{+}=S \theta^{-}, \quad S=\left[\begin{array}{ll}
0 & 1 \\
1 & 0
\end{array}\right] .
$$

The transition from one leg to another is assumed to take place in an infinitesimal length of time. Hence there exists no double support phase.

\subsection{A mixed logic dynamical model}

The model of a biped walker is hybrid in nature, consisting of continuous dynamics (single support phase) separated by abrupt changes (impact phase) of velocity. How to describe the hybrid system is very important which affects directly the synthesis of biped motion and the control performance.

For a general hybrid dynamical system, one may have several points of view: continuoustime, discrete-event, or mixed. Whether one of these manners applies better than the others depends a lot on the task. For the biped walking problem, we stress the walking motion transmissions, the adaptability to environment, and the robustness to unexpected disturbance. For that, we consider both continuous and discrete dynamics simultaneously including switching between different motion phases, express all the motions in a unified framework, which will allow the synthesis of the walking system in a systematic way.

Define the state variable vector as

$$
x=\left[\begin{array}{c}
\theta \\
\dot{\theta}
\end{array}\right]
$$

Then, the dynamical equation of single support phase (1) becomes

$$
\dot{x}=A_{c} x+B_{c}(t) \tau+b(t)
$$

where

$$
A_{c}=\left[\begin{array}{ll}
0 & 1 \\
0 & 0
\end{array}\right], \quad B_{c}(t)=\left[\begin{array}{c}
0 \\
M^{-1}(\theta)
\end{array}\right], \quad b(t)=\left[\begin{array}{c}
0 \\
-M^{-1}(\theta)(C(\theta, \dot{\theta}) \dot{\theta}+g(\theta))
\end{array}\right] .
$$

The transition equations of the angular velocity and joint angle, (3) and (4), become

$$
x^{+}=R(\alpha) x^{-}
$$

Where $x^{-}$is the pre-impact state, $x^{+}$is the post-impact state, and

$$
R(\alpha)=\left[\begin{array}{c}
S \\
\lambda(\alpha)
\end{array}\right]
$$

After impact, the biped robot progresses with switched pivot and its state evolves along the dynamical equation (6) again from the reset post-impact state of (7). The right side time derivation of the post-impact state satisfies the following relation.

$$
\begin{aligned}
\dot{x}^{+} & =A_{c} x^{+}+B_{c}\left(t^{+}\right) \tau+b\left(t^{+}\right) \\
& =A_{c} R(\alpha) x^{-}+B_{c}\left(t^{-}\right) \tau+\hat{b}\left(t^{-}\right),
\end{aligned}
$$


where

$$
B_{c}\left(t^{+}\right)=B_{c}\left(t^{-}\right), \quad \hat{b}\left(t^{-}\right)=\left[\begin{array}{c}
0 \\
-M^{-1}(2 \alpha)\left(C\left(S \theta^{-}, \lambda \dot{\theta}^{-}\right) \lambda \dot{\theta}^{-}+g\left(S \theta^{-}\right)\right)
\end{array}\right] .
$$

Here we introduce an auxiliary logic variable $\delta_{c}$ to associate the event of collision in order to describe (6) and (8) in a unified equation.

$$
\{\text { impact occurring }\} \leftrightarrow\left\{\delta_{c}=1\right\} \text {. }
$$

Then for both the single support phase and the impact phase, the state of the biped robot evolves monotonically along the following unified dynamical equation,

$$
\dot{x}=A_{c} x+B_{c}(t) \tau+A_{c}(R(\alpha)-I) z+(\hat{b}(t)-b(t)) \delta_{c}+b(t),
$$

where $z$ is the auxiliary variable defined as

$$
z=x \delta_{c} .
$$

It is remarkable that (11) can be equivalently expressed by a set of inequalities (Bemporad \& Morari, 1999),

$$
\begin{gathered}
0_{4 \times 1} \leq z \leq \delta_{c} \max (x), \\
x-\left(1-\delta_{c}\right) \max (x) \leq z \leq x .
\end{gathered}
$$

In practice, collision can be measured experimentally or predicted mathematically according to the toe position of swing leg. For a robot with two identical legs walking on smooth plane, a collision occurs if the joint angles satisfy the relation of $\theta_{1}=-\theta_{2}$. If $\theta_{1}+\theta_{2}>0$, it is the case of an interpenetration to the ground. For the convenience of numerical calculation, the condition of collision is practically represented as

$$
0 \leq \theta_{1}+\theta_{2} \leq \varepsilon_{2},
$$

where $\varepsilon_{2}$ is arbitrary small positive constant. The right side of the inequality is added to avoid taking deep interpenetration as collision.

In addition, the erected posture of robot, i.e. the case of $\theta_{1}=\theta_{2}=0$, has to be distinguished from collisions. Since the point of collision is always in front of the pivot in a nature walking, we set another condition for evaluating the occurrence of collision,

$$
\theta_{1}>0 \text {. }
$$

Concluding the previous two conditions, one can see that for a robot with two identical legs walking on smooth plane, the swing leg is in collision with the plane if the following linear inequalities are satisfied,

$$
\begin{gathered}
\varepsilon_{1} \leq \theta_{1}, \\
0 \leq \theta_{1}+\theta_{2} \leq \varepsilon_{2} .
\end{gathered}
$$

where $\varepsilon_{1}>0$. 
By using (13), we have more concrete expression for (9),

$$
\left\{\left\{\theta_{1} \geq \varepsilon_{1}\right\} \wedge\left\{\theta_{1}+\theta_{2} \leq \varepsilon_{2}\right\} \wedge\left\{\theta_{1}+\theta_{2} \leq 0\right\}\right\} \leftrightarrow\left\{\delta_{c}=1\right\} .
$$

The Boolean relation of (14) can be equivalently expressed by a set of linear inequalities. For that, we induce new logical variables $\delta_{1}, \delta_{2}, \delta_{3}$, which are associated with the following relations,

Consequently, we obtain

$$
\begin{gathered}
\left\{\theta_{1} \geq \varepsilon_{1}\right\} \leftrightarrow\left\{\delta_{1}=1\right\}, \\
\left\{\theta_{1}+\theta_{2} \leq \varepsilon_{2}\right\} \leftrightarrow\left\{\delta_{2}=1\right\}, \\
\left\{\theta_{1}+\theta_{2} \geq 0\right\} \leftrightarrow\left\{\delta_{3}=1\right\} .
\end{gathered}
$$

$$
\delta_{c}=\delta_{1} \delta_{2} \delta_{3}
$$

According to the known knowledge (Bemporad \& Morari, 1999), the equivalent inequalities of (15) are

$$
\begin{gathered}
\theta_{1} \geq \min \left(\theta_{1}\right)-\left(\min \left(\theta_{1}\right)-\varepsilon_{1}\right) \delta_{1}, \\
\theta_{1} \leq \varepsilon_{1}-\varepsilon_{0}+\left(\max \left(\theta_{1}\right)-\varepsilon_{1}+\varepsilon_{0}\right) \delta_{1},
\end{gathered}
$$

where $\varepsilon_{0}$ is arbitrary small positive constant. Similarly, the equivalent inequalities of (16) are

$$
\begin{gathered}
\theta_{1}+\theta_{2} \leq \varepsilon_{2}+\left(\max \left(\theta_{1}+\theta_{2}\right)-\varepsilon_{2}\right)\left(1-\delta_{2}\right), \\
\theta_{1}+\theta_{2} \geq \varepsilon_{2}+\varepsilon_{0}+\left(\min \left(\theta_{1}+\theta_{2}\right)-\varepsilon_{2}-\varepsilon_{0}\right) \delta_{2} .
\end{gathered}
$$

The equivalent inequalities of (17) are

$$
\begin{gathered}
\theta_{1}+\theta_{2} \geq \min \left(\theta_{1}+\theta_{2}\right)\left(1-\delta_{3}\right) \\
\theta_{1}+\theta_{2} \leq-\varepsilon_{0}+\left(\max \left(\theta_{1}+\theta_{2}\right)+\varepsilon_{0}\right) \delta_{3}
\end{gathered}
$$

It means that the joint angles $\theta_{1}$ and $\theta_{2}$ determinate the logical values of $\delta_{1}, \delta_{2}, \delta_{3}$ through the linear inequality set (18)-(20), thus the value of the impact flag $\delta_{c}=\delta_{1} \delta_{2} \delta_{3}$. However, the nonlinear relation $\delta_{c}=\delta_{1} \delta_{2} \delta_{3}$ requires special solver and complicates calculation. For this problem, we induce one more logical variable $\delta_{4}$ for variable linearization,

$$
\delta_{4}=\delta_{2} \delta_{3}
$$

By it the relation $\delta_{c}=\delta_{1} \delta_{2} \delta_{3}$ finally becomes

$$
\delta_{c}=\delta_{1} \delta_{4}
$$

The Boolean relation (21), (22) can be equivalently expressed by linear inequalities. For (21), the equivalent inequalities are

$$
\begin{aligned}
& -\delta_{2}+\delta_{4} \leq 0 \\
& -\delta_{3}+\delta_{4} \leq 0 \\
& \delta_{2}+\delta_{3}-\delta_{4} \leq 1
\end{aligned}
$$


For (23), the equivalent inequalities are

$$
\begin{aligned}
& -\delta_{1}+\delta_{c} \leq 0, \\
& -\delta_{4}+\delta_{c} \leq 0, \\
& \delta_{1}+\delta_{4}-\delta_{c} \leq 1 .
\end{aligned}
$$

Concluding the previous discussion, one can see that we get the unified dynamical equation (10) for the hybrid biped motion, which is subject to constraints of (2), (12), (18)-(20), (23), and (24). In the context, (10) is rewritten as (25). The constraints (2), (12), (18)-(20), (23), and (24) are integrated into (26).

$$
\begin{gathered}
\dot{x}=A_{c} x+B_{c}(t) \tau+B_{z}(t) z+B_{\delta}(t) \delta+b(t), \\
E_{1} x+E_{2} \tau+E_{3} z+E_{4} \delta \leq E_{5},
\end{gathered}
$$

where

$$
\delta=\left[\begin{array}{lllll}
\delta_{c} & \delta_{1} & \delta_{2} & \delta_{3} & \delta_{4}
\end{array}\right]^{T}, \quad B_{z}(t)=A_{c}(R(\alpha)-I), \quad B_{\delta}(t)=\left[\begin{array}{llllll}
\hat{b}(t)-b(t) & 0 & 0 & 0 & 0
\end{array}\right] .
$$

The coefficient $E_{i}$ of (26), $i=1, \ldots, 5$, can be known from the context.

For computational convenience, (25) is generally put in discrete-time domain representation.,

$$
x(k+1)=A x(k)+B_{1}(k) \tau(k)+B_{2}(k) z(k)+B_{3}(k) \delta(k)+B_{0}(k),
$$

where with the sampling period $T_{s}$,

$$
\begin{gathered}
A=e^{A_{c} T_{s}}, \quad B_{1}(k)=\int_{0}^{T_{s}} e^{A_{c}\left(T_{s}-t\right)} d t \cdot B_{c}(k), \quad B_{2}(k)=\int_{0}^{T_{s}} e^{A_{c}\left(T_{s}-t\right)} d t \cdot B_{z}(k), \\
B_{3}(k)=\int_{0}^{T_{s}} e^{A_{s}\left(T_{s}-t\right)} d t \cdot B_{\delta}(k), \quad B_{0}(k)=\int_{0}^{T_{s}} e^{A_{s}\left(T_{s}-t\right)} d t \cdot b(k) .
\end{gathered}
$$

The MLD model of (25), (26) or (27), (26) describes both the continuous dynamics and the impact event within one framework, which provides the possibility of systematic synthesis and control for the biped motion.

\section{Progressing constraint}

A successful walking should be stable and successive progress forward. For that, conditions of stable and successive walking have to be taken into account as constraints subject to the optimal gait generation problem.

\subsection{Erected body}

For supporting the body against gravity, the position of hip should be above a positive level to avoid falling. For example, to achieve

$$
\ell^{*} \leq y_{\text {hip }} \leq \ell,
$$


where $y_{\text {hip }}$ is the vertical position of hip, we can set

$$
-\theta_{1}^{*} \leq \theta_{1} \leq \theta_{1}^{*}
$$

With $\theta^{*}=\arccos \ell^{*} / \ell$.

\subsection{Progression}

For propel the body in the intended direction with a progression rhythm, horizontal velocity of hip should be kept positive, and strictly monotonically increased. The horizontal position of hip is

$$
x_{\text {hip }}=\ell \sin \theta_{1} \text {. }
$$

Its time derivation is the horizontal velocity of hip, which should satisfy

$$
\dot{x}_{h i p}=\dot{\theta}_{1} \ell \cos \theta_{1}>0 \text {. }
$$

For that, we set the following constraint to ensure the forward progression of the body.

$$
\dot{\theta}_{1} \geq \varepsilon_{v}
$$

where $\varepsilon_{v}>0$.

\subsection{Environment}

For walking at non-smooth ground, we suppose that the environment at the next steps can be detected by sensor such as camera. Then the non-smooth walking surface can be mathematically expressed by a set of equalities, denoted as

$$
y=\psi(x) \text {. }
$$

Note that in this case, the occurrence condition of collision, $y_{t o e}=\psi(x)$, has to be derived to substitute for (13). Corresponding with this, within single support phase, the toe of the swing leg has to be controlled above the walking surface,

$$
y_{\text {toe }}>\psi(x) \text {. }
$$

These control constraint (28)-(30) can be integrated together with (26) which is the constraint part of the MLD model of the biped robot. The integrated MLD model of (25), (26), (28)-(30) is the unified description for the biped motion, including both the physical modeling and the motional requirement.

\section{Optimal control}

The problem is to generate on line the biped motion without pre-defined trajectory, by the simultaneous synthesis and control of walking gait. The optimal gait to current environment consists of the continuous trajectory within each single support phase and the transition time of stance leg. 
Basing on our derived MLD model, we handle the control objective by using a systematical method such as the model predictive control (MPC). Note that traditionally, MPC is used to solve the control problem of a constrained but continuous dynamical system. For the biped motion control, because of the existence of logical variables in the MLD model, the MPC of the MLD system is more complex. The idea is to carry out optimization of the biped motion in a receding finite horizon, which can be transformed to solve a mixed integer quadric programming (MIQP) problem (Bemporad and Morari, 1999). Its solution corresponds to the optimal walking gait of the biped robot.

The criterion for minimization is as the following.

$$
L=\sum_{i=0}^{N-1}\left(\left\|x(k+i+1 \mid k)-x_{r}\right\|_{Q_{1}}+\|\tau(k+i)\|_{Q_{2}}+\left\|z(k+i)-z_{r}\right\|_{Q_{3}}+\left\|\delta(k+i)-\delta_{r}\right\|_{Q_{4}}\right)
$$

which is subject to the MLD model of (27), (26), (28)-(30). In (31), the real number $N$ is the horizon for each minimization. $x(k+i+1 \mid k)$ is the state in the $i+1$ steps future of the current state $x(k)$, and can be predicted by the MLD model according to the applied torque sequence $\tau(k), \ldots, \tau(k+i) . x_{r}$ is the desired state at preimpact point, can be time invariant or time-varying. $z_{r}$ is the corresponding auxiliary continuous variable of $x_{r} \cdot \delta_{r}$ is the corresponding auxiliary logical variable of $x_{r} \cdot Q_{1}, \ldots, Q_{4}$ are the weighting matrices.

The first term in (31) is for achieving a progressive walking. In the $N$ steps optimization, the state before impact will be driven to approach the impact point $x_{r}$. The state post or after impact will be driven to access the next impact point $x_{r}$ in order to keep progression. It implies that the criterion (31) and the MLD model can handle the impact effect well even if the impact point appears as an interior point of the optimization, and its solution is optimal for both before impact and after impact state. The second term in (31) is for achieving a minimal torque control purpose.

Note that (27) is a nonlinear MLD model in the sense that it has nonlinear and time-varying coefficients such as $B_{2}(k)$. However, the optimization of a nonlinear MLD system is difficult to solve because of the system nonlinearity and the curse of dimension. In this study, we linearize the nonlinear MLD system so as to avoid the computational difficulty. The linearization is carried out by freezing the coefficient matrices at the current values $x(k)$ for the next $N$ steps. Basing on the linearized MLD system, we can solve its MPC problem by using a MIQP solver to get the future $N$ steps control inputs $\tau(k), \ldots, \tau(k+N-1)$. Then, we apply only the first control input $\tau(k)$ to the actual nonlinear mechanical system for one sampling period, which results in a new state $x(k+1)$. At this updated working point, the above linearization and optimal calculation are repeated for keeping control.

The control procedure is concluded as follows.

1. Set the sampling period $T_{s}$ and the horizon $N$ for optimization.

2. Substituting the current state $x(k)$ into the nonlinear MLD model (27), and freezing the coefficient matrices at the current values for the next $N$ steps.

3. Based on the linearized MLD model, solve the MPC problem by MIQP solver. 
4. Select only the control input for the next step, and apply it to the nonlinear biped robot. Get the updated state $x(k+1)$.

5. The time is shift from $k$ to $k+1$. Then repeat the step 2-5.

The proposed linearization and the MPC are based on the updating state. This feedback brings robust property to the control system, by which the nonlinear model error and external disturbance can be effectively treated. On the other hand, larger number $\mathbf{N}$ results in better control performance. But it increases the number of variables and leads the optimal problem more complex. Especially, the number of logical variables determinates the computational time exponentially. The computational burden will obstruct the real time implementation of the control. For this problem, the use of lookup table and the technique of mp-MIQP (Bemporad et al., 2002), which moves the complex computation to off line work, will drastically reduce the on line processing time.

\section{Application to a biped walker system}

The proposed MLD modeling and MPC approach are applied to a 2 D.O.F. planar biped robot system as shown in Fig.1. The physical parameters of the biped robot are given in Table 1.

\begin{tabular}{|l|l|l|l|}
\hline$m_{h}$ & $m$ & $\ell$ & $r_{g}$ \\
\hline $10 \mathrm{~kg}$ & $5 \mathrm{~kg}$ & $1 \mathrm{~m}$ & $0.5 \mathrm{~m}$ \\
\hline
\end{tabular}

Table 1. Physical parameters of the 2 D.O.F. biped walker

For simplicity, the walking ground is assumed to be smooth plane. The motion of the biped is the automatic transition of single support phase and impact phase. The movement range of link 1 and link 2 is set within $\pm \pi / 4$ (rad) which ensures the position of hip above $0.7 \mathrm{~m}$ to the ground. The torque limitation of link 1 is supposed to be $100(\mathrm{Nm})$, and that of link 2 is $30(\mathrm{Nm})$. The minimal progressing velocity of hip, $\varepsilon_{v}$, is set to be $0.1(\mathrm{rad} / \mathrm{s})$.

$\uparrow(6 \mathrm{pt})$

In our simulations, the biped robot started to walk with an initial state

$$
x_{0}=\left[\begin{array}{llll}
-0.22 & 0.22 & 0.8 & -0.4
\end{array}\right]^{T} .
$$

To carry out the MPC for generating the walking gait optimally, a reference pre-impact state is required as stated in (31), which is taken as

$$
x_{r}=\left[\begin{array}{llll}
0.23 & -0.23 & 1.30 & 1.63
\end{array}\right]^{T} .
$$

The corresponding auxiliary variables are

$$
\begin{aligned}
z_{r} & =\left[\begin{array}{llll}
0.23 & -0.23 & 1.30 & 1.63
\end{array}\right]^{T}, \\
\delta_{r} & =\left[\begin{array}{llll}
1 & 1 & 1 & 1
\end{array}\right]^{T} .
\end{aligned}
$$

The weighting matrix is chosen to be

$$
Q_{1}=\operatorname{diag}\left(10^{-4}, 10^{-4}, 10^{-7}, 10^{-7}\right) .
$$


The other weighting matrices $Q_{2}, Q_{3}, Q_{4}$ are appointed to be diagonal matrices, and all of their diagonal elements are equal to $10^{-10}$.

All simulations are executed by a computer with a Pentium 3.20GHz CPU and 3G memory. The calculation for the synthesis of walking motion is carried out using MATLAB. The sampling period $T_{s}$ is $10 \mathrm{~ms}$.

The first simulation is for the synthesis of biped motion. The simulation results are shown in Fig.2 and Fig.3. In Fig.2(a), the real line is the trajectory of joint $\theta_{1}(t)$, and the dot line is the trajectory of joint $\theta_{2}(t)$. In Fig.2(b), the real line is the trajectory of the angular velocity $\dot{\theta}_{1}(t)$, and the dot line is the trajectory of the angular velocity $\dot{\theta}_{2}(t)$. In Fig.3(a), it shows the profile of the applied torque $\tau_{1}$. In Fig.3(b), it is the profile of the applied torque $\tau_{2}$.

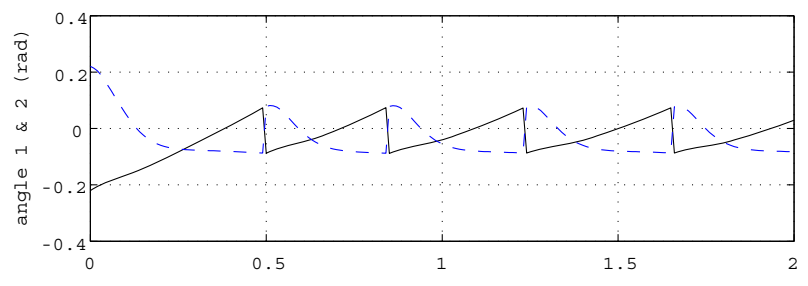

(a) time (s)

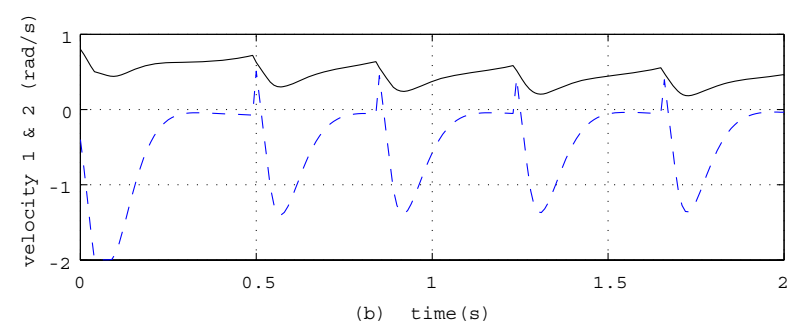

Figure 2. The time histories of joint angle and velocity: (a) the angle of support leg, $\theta_{1}$ (rad) (the real line) and the angular of swing leg, $\theta_{2}$ (rad) (the dot line). (b) angular velocity of support leg, $\dot{\theta}_{1}(\mathrm{rad} / \mathrm{s})$ (the real line) and angular velocity of swing leg, $\dot{\theta}_{2}(\mathrm{rad} / \mathrm{s})$ (the dot line)

From these Fig. 2 and Fig.3, one can see that the biped robot is controlled to progress 5 steps in $2 \mathrm{~s}$. The first step period is $0.5 \mathrm{~s}$, slower than the left 4 steps. Leg transition occurred at the position of less than the desired $0.23(\mathrm{rad})$. Thus the stride is relative small.

The second simulation is to check the robust property of the approach. For that, a pulse type of disturbance vector $w=\left[\begin{array}{ll}4 & 10\end{array}\right]^{T}$ is added to the torque at $1 \mathrm{~s}$. The profiles of the disturbed torques are shown in Fig.4. From Fig.4 (a) and (b), it is seen that both of the torques converge to their stable trajectories after the disturbance disappeared. The trajectories of angles and angular velocities have not big change from those shown in Fig.2.

By these simulation results, we see that the MLD approach is effective for generating gait pattern, and is robust to external disturbance. However, the computation time for both simulations is about $88 \mathrm{~s}$ for the $2 \mathrm{~s}$ walking. Note that these simulations are preliminary studies for validating the effectiveness of MLD modeling and the feasibility of MPC, the computation time is absolutely not optimized and the used MIQP solver is not fast. This 
provides us another important research topic of how to decrease the computation time for real time implementation.

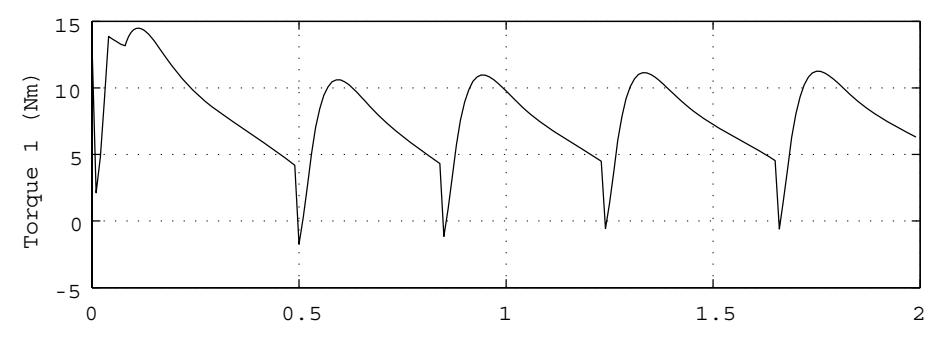

(a) time (s)

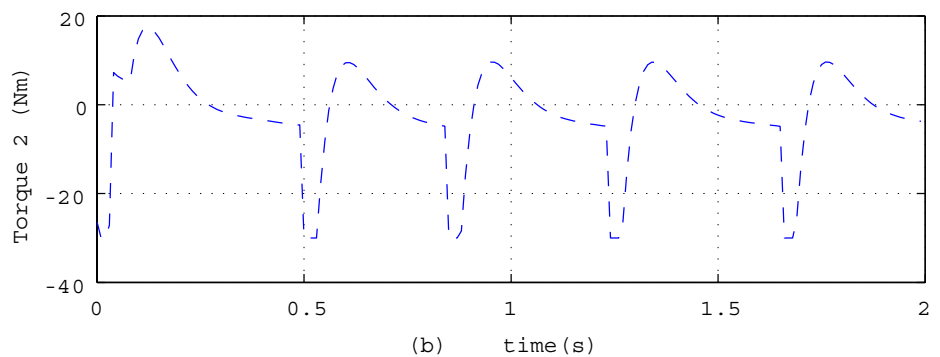

Figure 3. The profiles of torques: (a) torque $\tau_{1}(\mathrm{Nm})$ of the support leg, (b) torque $\tau_{2}(\mathrm{Nm})$ of the swing leg
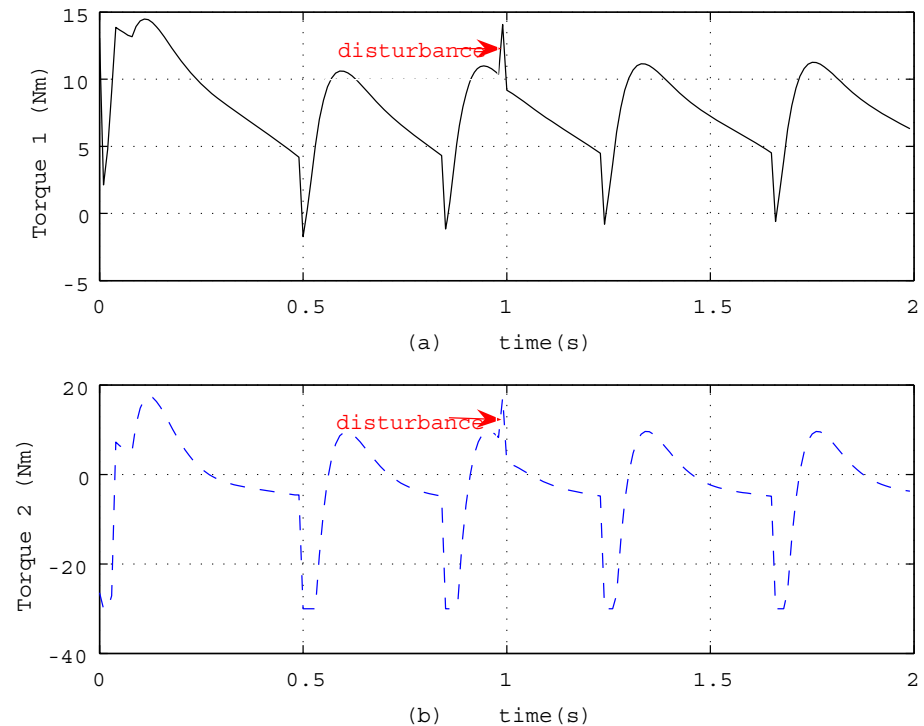

Figure 4 . The profiles of the disturbed torques: (a) torque $\tau_{1}(\mathrm{Nm})$ of the support leg, (b) torque $\tau_{2}(\mathrm{Nm})$ of the swing leg 


\section{Conclusion}

In this study, we proposed a MLD modeling and MPC approach for the on line optimization of biped motion. Such modeling approach possesses advantage that it describes both the continuous dynamics and the impact event within one framework, consequently it provides a unified approach for mathematical, numerical and control investigations. This MLD model allows model predictive control (MPC) and subsequent stability from the numerical analysis viewpoints, by powerful MIQP solver. Hence the biped robot can be on line controlled without pre-defined trajectory. The optimal solution corresponds to the optimal gait for current environment and control requirement. The feasibility of the MLD model based predictive control is shown by simulations. How to effectively decrease the computation time in order to realize the real time implementation is an important research topic left to future.

Finally, we mention that a human uses his predictive function based on an internal model together with his feedback function for motion, which is considered as a motor control model of a cerebellum (Kawato, 1999). Stimulated by this, a general theoretical study for motion control of hybrid systems is reported in (Yin \& Hosoe, 2004) which is based on the MLD model of a hybrid system. We are further developing this theory to help the biped motion synthesis and control. It will be also useful for the realization of complex motion of other bio-mimetic robots.

\section{References}

Azevedo, C., Poignet, P. \& Espiau, B. (2004). Artificial locomotion control: from human to robots, Robotics and Autonomous Systems, Vol.47, Issue 4, (July 2004) pp.203-223, ISSN 0921-8890.

Bemporad, A. \& Morari, M. (1999). Control of systems integrating logic, dynamics, and constraints, Automatica, Vol.35, No.3, (March 1999) pp.407-427, ISSN 0005-1098.

Bemporad, A., Morari, M., Dua, V. \& Pistikopoulos, E.N. (2002). The explicit linear quadratic regulator for constrained systems, Automatica, Vol.38, No.1, (January 2002) pp.3-20, ISSN 0005-1098.

Chevallereau, C., Formalapos sky, A. \& Perrin, B. (1998). Low energy cost reference trajectories for a biped robot, Proceedings of the IEEE International Conference on Robotics and Automation (ICRA), pp.1398-1404, ISBN 0-7803-4300-X, Leuven, Belgium, May 1998, IEEE.

Chevallereau, C. \& Sardain, P. (2000). Design and actuation optimization of a 4 axes biped robot for walking and running, Proceedings of the IEEE International Conference on Robotics and Automation (ICRA), pp.3365-3370, ISBN 0-7803-5886-4, San Francisco, CA, USA, April 2000, IEEE.

Chew, C.M. \& Pratt, G.A. (2000). A general control architecture for dynamic bipedal walking, Proceedings of the IEEE International Conference on Robotics and Automation (ICRA), pp.3989-3995, ISBN 0-7803-5886-4, San Francisco, CA, USA, April 2000, IEEE.

Denk, J. \& Schmidt, G. (2001). Walking primitive synthesis for an anthropomorphic biped using optimal control techniques, Proceedings of the International Conference on Climbing and Walking Robots (CLAWAR), pp.819-826, ISBN 1-86058-265-2, Karlsruhe, Germany, September 2001, Professional Engineering Publishing, Bury St Edmunds (u.a.), UK. 
Kawato, M. (1999). Internal models for motor control and trajectory planning, Current Opinion in Neurobiology, Vol.9, No.6, (December 1999) pp.718-727, ISSN 0959-4388.

Kooij, H. van der, Jacobs, R., Koopman, B. \& Helm, F. van der (2003). An alternative approach to synthesizing bipedal walking, Biological Cybernetics, Vol.88, No..1, (January 2003) pp.46-59, ISSN 0340-1200.

Park, J.H. \& Chung, H. (1999) . Zmp compensation by on-line trajectory generation for biped robots, Proceedings of the IEEE Conference on Systems, Man and Cybernetics (SMC99), pp.960-965, ISBN 0-7803-5731-0, Tokyo, Japan, October 1999, IEEE.

Park, J.H. \& Chung, H. (2000). Hybrid control of biped robots to increase stability in locomotion, Journal of Robotic Systems, Vol.17, Issue 4, (Published online: March 2000) pp.187-197, ISSN 0741-2223.

Yin, Y.J. \& Hosoe, S. (2004). Tracking Control of Mixed Logic Dynamical Systems, IEICE Trans. on Fundamentals of Electronics, Communications and Computer Sciences, Vol.E87A, No.11, (November 2004) pp.2929-2936, ISSN 09168508. 


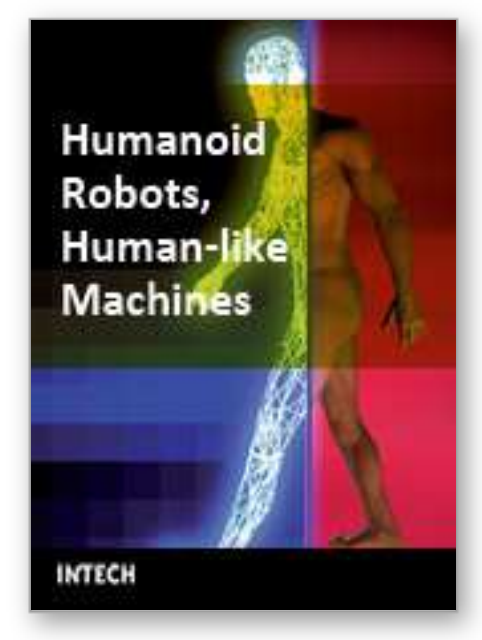

\author{
Humanoid Robots, Human-like Machines \\ Edited by Matthias Hackel
}

ISBN 978-3-902613-07-3

Hard cover, 642 pages

Publisher I-Tech Education and Publishing

Published online 01, June, 2007

Published in print edition June, 2007

In this book the variety of humanoid robotic research can be obtained. This book is divided in four parts: Hardware Development: Components and Systems, Biped Motion: Walking, Running and Self-orientation, Sensing the Environment: Acquisition, Data Processing and Control and Mind Organisation: Learning and Interaction. The first part of the book deals with remarkable hardware developments, whereby complete humanoid robotic systems are as well described as partial solutions. In the second part diverse results around the biped motion of humanoid robots are presented. The autonomous, efficient and adaptive two-legged walking is one of the main challenge in humanoid robotics. The two-legged walking will enable humanoid robots to enter our environment without rearrangement. Developments in the field of visual sensors, data acquisition, processing and control are to be observed in third part of the book. In the fourth part some "mind building" and communication technologies are presented.

\title{
How to reference
}

In order to correctly reference this scholarly work, feel free to copy and paste the following:

Yingjie Yin and Shigeyuki Hosoe (2007). Mixed Logic Dynamical Modeling and On Line Optimal Control of Biped Robot, Humanoid Robots, Human-like Machines, Matthias Hackel (Ed.), ISBN: 978-3-902613-07-3, InTech, Available from:

http://www.intechopen.com/books/humanoid_robots_human_like_machines/mixed_logic_dynamical_modeling _and_on_line_optimal_control_of_biped_robot

\section{INTECH}

open science | open minds

\section{InTech Europe}

University Campus STeP Ri

Slavka Krautzeka 83/A

51000 Rijeka, Croatia

Phone: +385 (51) 770447

Fax: +385 (51) 686166

www.intechopen.com

\section{InTech China}

Unit 405, Office Block, Hotel Equatorial Shanghai

No.65, Yan An Road (West), Shanghai, 200040, China

中国上海市延安西路65号上海国际贵都大饭店办公楼 405 单元

Phone: +86-21-62489820

Fax: +86-21-62489821 
(C) 2007 The Author(s). Licensee IntechOpen. This chapter is distributed under the terms of the Creative Commons Attribution-NonCommercial-ShareAlike-3.0 License, which permits use, distribution and reproduction for non-commercial purposes, provided the original is properly cited and derivative works building on this content are distributed under the same license. 